\title{
ANISOTROPIC LINE EMISSION AND THE GEOMETRY OF THE BROAD-LINE REGION IN ACTIVE GALACTIC NUCLEI
}

\author{
G. J. Ferland, ${ }^{1}$ B. M. Peterson,${ }^{1}$ K. Horne, ${ }^{2}$ W. F. Welsh, ${ }^{1,2,3}$ and S. N. Nahar ${ }^{1}$ \\ Received 1991 June 3; accepted 1991 September 4
}

\begin{abstract}
The rapid response of the broad emission lines to continuum variations in the Seyfert galaxy NGC 5548 implies that line-emitting regions are very close to the continuum source. As described in this contribution, photoionization models must be modified to deal with the very high radiation densities in the broad-line region. An immediate consequence of the high radiation density is that the emission-line cloud column densities must be large in order for the clouds to remain optically thick in the ionizing continuum and hence show recombination line variability. This leads to significant optical depths in the lines as well, with the result that line emission is radiated anisotropically, with most of the radiation emitted from the inner, irradiated face of the cloud. This may account for the apparent absence of line-emitting material along our line of sight to the continuum source, as inferred from reverberation studies. We show that the observed continuum- $\mathrm{H} \beta$ transfer function for NGC 5548 is consistent with a spherical distribution of anisotropically radiating clouds.

We estimate the density in the Ly $\alpha-C$ IV-emitting regions as $N \approx 10^{11} \mathrm{~cm}^{-3}$, largely due to the lack of significant C III] $\lambda 1909$ emission from this gas. The ionization parameter is then set by the source-cloud separation. The clouds are predicted to be significant emitters of $O \vee \lambda 1218$, which would cause Ly $\alpha$ to appear redshifted and respond in a nonlinear manner. We show that clouds at slightly higher densities but cosmic abundances can be strong emitters of lines such as $\mathrm{Al}$ III $\lambda 1860$, largely because cooling by other lines is suppressed by line thermalization at these densities.
\end{abstract}

Subject headings: atomic processes - galaxies: individual (NGC 5548) - galaxies: nuclei galaxies: Seyfert - line formation — quasars: emission lines

\section{INTRODUCTION}

Emission-line-continuum reverberation studies have revealed that broad-line region (BLR) clouds of active galactic nuclei (AGNs) are exposed to a far more intense radiation field than previously thought (see the review by Peterson 1988). The implications of this radiation field are several (Ferland \& Persson 1989): (1) the cloud equation of state of "conventional" density clouds $\left(N \approx 10^{9} \mathrm{~cm}^{-3}\right)$ is dominated by line radiation pressure, so that the geometry is unstable unless self-gravitating; (2) carbon and oxygen "Strömgren spheres" form when the radiation density is large, so that the heavy element line spectrum is largely insensitive to many parameters (which may explain why AGN emission-line spectra are so similar; and (3) nearly all emission lines are optically thick, and most are observed against a continuum (usually Balmer or Paschen) which is itself optically thick.

The fact that the radiation density is very large at the BLR radius was made clear in the review by Peterson. This conclusion is verified by the recent intensive study of NGC 5548 (Clavel et al. 1991; Peterson et al. 1991). The radiation density at the $\mathrm{Ly} \alpha-\mathrm{C}$ IV $\lambda 1549$ radius corresponds to an ionization parameter of $\log (U) \approx 0.5$ for conventional BLR densities, more than two orders of magnitude larger than the "standard" value (Davidson \& Netzer 1979). Another result is that there is no clear density indicator for the $\mathrm{Ly} \alpha-\mathrm{C} \mathrm{IV}$ region, since the $\mathrm{C}$ III] $\lambda 1909$ is formed at a distance of $\sim 3-4$ lt-weeks.

\footnotetext{
${ }^{1}$ Department of Astronomy, The Ohio State University, 174 West 18th Avenue, Columbus, $\mathrm{OH} 43210$.

${ }^{2}$ Space Telescope Science Institute, 3700 San Martin Drive, Baltimore, MD 21218.

${ }^{3}$ Postal address: Space Telescope Science Institute.
}

The line-continuum reverberation measurements can be transformed into a "map" of the emission-line regions if the response of a given line to changes in the continuum, and the line "beaming function" (i.e., the fraction of the line which is beamed toward the source of ionizing radiation, as opposed to being isotropically radiated) is known (Blandford \& McKee 1982). In this contribution, we outline an escape probabilitybased formalism, including the interaction with a background opacity, which is used to predict line-beaming factors for BLR clouds (Appendix B). We also outline several computational details necessary to model gas exposed to an intense nonthermal continuum (Appendix A). We find that all hydrogen lines are strongly inwardly beamed, and that C IV $\lambda 1549$ is moderately beamed. We then discuss the implications of this for the geometry of the emission-line regions of AGNs; beaming causes the source-cloud separation to be overestimated by variability studies (so that the radiation field at the BLR is even more intense than previously thought) and can cause even spherical geometries to have little instantaneous response to continuum changes.

\section{THE CASE OF NGC 5548}

Here we determine a set of fiducial conditions for "typical" clouds in NGC 5548, a well-studied Seyfert galaxy. We note explicitly that it is not our intent to model the emission-line spectrum of this object in any detail at this time. The data on NGC 5548 show very clearly that radial dependence of the emissivity of each line is unique, and a realistic calculation will take this into account. For the sake of simplicity, we will consider the behavior only of representative BLR clouds at a representative distance from the ionizing source. 


\subsection{The BLR Radius}

Variability studies show that the BLR is highly stratified (Clavel et al. 1991; Peterson et al. 1991). Emission-line response time scales range between essentially zero for $\mathrm{He}$ II $\lambda 1640$ and up to several weeks for lower ionization lines such as C III] $\lambda 1909$. The situation is further complicated by the fact that the lag (i.e., the phase shift between the continuum and emission-line light curves) apparently depends on the details of the outburst; Netzer \& Maoz (1990) show that the Ly $\alpha$ lag ranges between a few days and about 20 days, depending on which of the three outbursts detected in the Clavel et al. study is used.

Clearly the situation is complex. We adopt 8 lt-days as a "typical" BLR radius, since this is close to the mean of the lag determined for the two strongest emission lines in the Clavel et al. (1991) data set, C Iv $\lambda 1549$ and Ly $\alpha$. We have deliberately chosen to err in the direction of a small BLR radius, since as we will show in $\S 4$, in the case of anisotropic line emission, the continuum-emission-line lag tends to overestimate the BLR size as we see primarily the far side of the BLR.

\subsection{The Flux of Ionizing Photons}

Here we estimate the flux of ionizing photons at the C IVLy $\alpha$ radius in NGC 5548. This is then used to estimate the ionization parameter, the parameter of interest to models of the emission-line regions.

Clavel et al. (1991) find a rest frame mean flux at $1315 \AA$ of $\lambda f_{\lambda}(1315 \AA)=6.06 \times 10^{-11} \mathrm{ergs} \mathrm{cm} \mathrm{cm}^{-2} \mathrm{~s}^{-1}$, corresponding to $v L_{v}(1315 \AA)=1.8 h^{-2} \times 10^{43}$ ergs $\mathrm{s}^{-1}$, where $h$ is the Hubble constant in units of $100 \mathrm{~km} \mathrm{~s}^{-1} \mathrm{Mpc}^{-1}$. (When specific quantities are evaluated below, we shall assume $h=0.75$.) Similarly, the X-ray continuum reported by Turner \& Pounds (1989) corresponds to $v L_{v}(2-10 \mathrm{keV})=1.0 h^{-2} \times 10^{43} \mathrm{ergs} \mathrm{s}^{-1}$. Both the UV and $X$-ray continua are highly variable; within the considerable latitude granted by variability, the general shape of the continuum is consistent with the mean AGN continuum derived by Mathews \& Ferland (1987). Numerically integrating over the "generic" radio-quiet AGN continuum of Mathews \& Ferland (1987), normalized to the flux at $1350 \AA$, we find a total number of hydrogen ionizing photons of

$$
Q(\mathrm{H})=1.0 h^{-2} \times 10^{54} \mathrm{~s}^{-1}
$$

with an uncertainty of $\sim 0.5$ dex due to both the uncertainty in $h$ and the shape of the extreme ultraviolet continuum. The corresponding luminosity in ionizing photons is $6.6 h^{-2} \times 10^{43}$ ergs $\mathbf{s}^{-1}$.

Taking 8 lt-days $\left(2 \times 10^{16} \mathrm{~cm}\right)$ as typical of the separation between the continuum source and BLR clouds, then the flux of ionizing photons is

$$
\phi(\mathrm{H})=\frac{Q(\mathrm{H})}{4 \pi r^{2}} \approx 1.9 h^{-2} \times 10^{20} \mathrm{~cm}^{-2} \mathrm{~s}^{-1} .
$$

Throughout this work we shall refer to an ionization parameter which is the dimensionless ratio of ionizing photon to baryon densities:

$$
U \equiv \frac{\phi(\mathrm{H})}{c N_{\mathrm{H}}},
$$

where $c$ is the speed of light, and $N_{\mathrm{H}}$ is the number density of hydrogen in all forms. $N_{\mathrm{H}}$ is not known for the C IV-Ly $\alpha$ region; conventional models were constrained to densities below $N \sim 10^{10} \mathrm{~cm}^{-3}$ by the presence of $\mathrm{C} \mathrm{III]} \lambda 1909$ (Davidson \& Netzer 1979) but it is now known that the C III] line is formed at much larger radii than the higher ionization lines (Clavel et al. 1991). Determining the density of the gas at 8 lt-days is a major goal of the following section. It is generally taken to be in the neighborhood of $N_{\mathrm{H}} \approx 10^{9.5} \mathrm{~cm}^{-3}$. The value of the ionization parameter corresponding to the flux of ionizing photons is $\log \left(U \times N_{10}\right) \approx 0$, where $N_{10}$ is the density in units of $10^{10} \mathrm{~cm}^{-3}$.

\section{PHOTOIONIZATION CALCULATIONS}

Here we present photoionization calculations which extend the work of Ferland \& Persson (1989) to the radiation densities suggested by recent reverberation studies of NGC 5548.

\subsection{Dependence on the Submillimeter Break}

The incident continuum used here is based on that inferred by Mathews \& Ferland (1987). The energy of the submillimeter break in the incident continuum has a major effect on the calculations, largely because of the strong free-free heating which results. We show that the importance of this heating mechanism increases with the intensity of the incident radiation field.

In photoionization equilibrium, the photoelectric heating rate at the illuminated face of the cloud is proportional to $N^{2} \alpha\left\langle h v-E_{0}\right\rangle$ ergs $\mathrm{cm}^{-3} \mathrm{~s}^{-1}$, where $\alpha$ is the recombination coefficient, $N$ is the number density, $E_{0}$ is the threshold energy for $\mathrm{H}$ ionization, and $\left\langle h v-E_{0}\right\rangle$ is the mean energy of a photoelectron. The heating rate has no direct dependence on the ionization parameter or photon flux, although the gas does tend to get hotter for more intense radiation fields as the level of ionization increases and the ability of the gas to cool effectively diminishes.

This is to be contrasted with free-free heating (Ferland \& Persson 1989). Here the local heating rate is proportional to the intensity of the continuum at energies where the gas absorbs effectively; $\Lambda_{v} \sim f_{v} \sigma_{v}$. The interplay between photoelectric and free-free absorption heating is intricately related to the temperature of the gas and the shape of the radiation field and is best illustrated by a complete calculation.

Figure 1 shows the results of a set of calculations in which the intensity of the incident radiation field at ionizing energies was held fixed at the value quoted above (eq. [2]). The hydrogen density was also held fixed at $N_{\mathrm{H}}=10^{10} \mathrm{~cm}^{-3}$, and the wavelength of the submillimeter break was varied between 1 and $1000 \mu \mathrm{m}$. The incident continuum is that given by Mathews \& Ferland (1987), with the one exception that the spectral index is changed from -1 to $+5 / 2$ for energies lower than the break, i.e., $f_{v} \propto v^{5 / 2}$ at low energies. This is appropriate for self-absorbed synchrotron emission.

The upper panel of Figure 1 shows that the temperature at the illuminated face of the cloud rises sharply when the wavelength of the break increases longward of $\sim 50 \mu \mathrm{m}$. The lower panel shows the intensities of two strong cooling lines relative to $\operatorname{Ly} \alpha$. The intensity of $\operatorname{Ly} \alpha$ is proportional to the recombination rate, so the line ratios are proportional to the cooling per recombination, or (since the clouds are assumed to be in steady state) the heating per photoionization. The relative line ratios increase when free-free heating increases because this process, unlike those involving more energetic photons, produces heating without ionization.

In the calculations below we assume a break at $100 \mu \mathrm{m}$. This nearly reproduces the observed intensity of C IV $\lambda 1549 / \mathrm{Ly} \alpha$. 


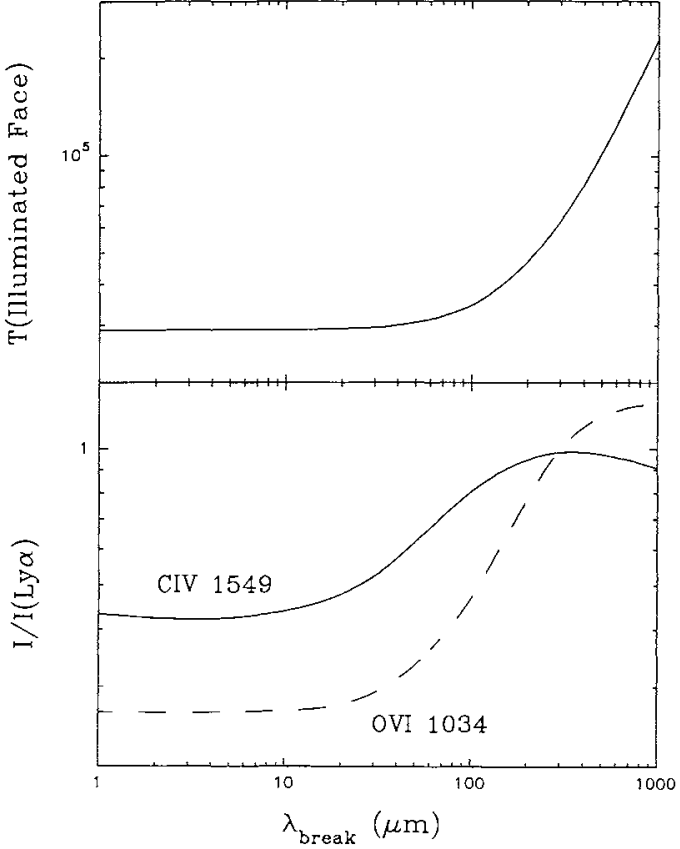

Fig. 1.-The effect of the submillimeter continuum on the C IV-Ly $\alpha$ clouds in NGC 5548 is shown. The temperature at the illuminated face of a cloud 8 It-days from the continuum source is shown as a function of the assumed submillimeter break in the upper panel of the figure. The lower panel shows the intensities of the C IV 21549 and $O$ VI 21034 lines relative to Ly $\alpha$. Free-free heating becomes progressively more important when the submillimeter break occurs longward of $\sim 50 \mu \mathrm{m}$. These lines become stronger relative to Ly $\alpha$ as the amount of heating contributed by the IR continuum increases.

\subsection{Dependence on the Hydrogen Density}

The line transfer functions for C III] $\lambda 1909$ and C IV $\lambda 1549$ (Krolik et al. 1991) show that the emissivity distributions for these two lines are not the same; the transfer function of $\mathrm{C}$ IV peaks near $\tau=8$.days, while that of $\mathrm{C} \mathrm{III]} \mathrm{is} \mathrm{increasing} \mathrm{at} \tau=8$ days toward a peak near 25 days. An immediate consequence of this is that there is no clear density diagnostic indicator for the BLR gas. In principle, the C III $\lambda 1909 / C$ IV $\lambda 1549$ flux ratio can be determined as a function of time delay from the ratio of the $\mathrm{C}$ III] and $\mathrm{C}$ IV transfer functions, although one must bear in mind that this applies only to the variable components of the two lines; the situation is further complicated by the possibility of anisotropic line emission, as discussed in the following sections, since the transformation from time delay to radial distribution becomes more complicated. Determination of the C III] $\lambda 1909 / \mathrm{C}$ Iv $\lambda 1549$ flux ratio from the transfer functions derived from real data is a major source of uncertainty since the transfer functions themselves are not well resolved, and the $\mathrm{C} \mathrm{III]} \lambda .1909$ light curve is relatively noisy. In particular, the amplitude of the function away from the peak may be artificially too high.

Bearing these difficulties in mind, we will nevertheless use the relative amplitudes of the $C_{\text {III }} \lambda 1909$ and $C_{\text {IV }} \lambda 1549$ transfer functions from Krolik et al. (1991), weighted by the respective total broad-component fluxes, to estimate the C III] $\lambda 1909 / \mathrm{C}$ IV $\lambda 1549$ flux ratio as $\sim 0.1$ at 8 lt-days from the continuum source. A more accurate treatment would require a complete specification of the radial emissivity distribution for each line and determination of whether or not the resulting transfer functions are consistent, within the uncertainties, with the observational realizations of the transfer functions. This work is under way, but is beyond the scope of the present contribution.

Figure 2 shows the results of a series of calculations using the continuum described above, a Lyman-continuum optical depth of $10^{4}$, and various hydrogen densities. Line ratios are shown relative to Ly $\alpha$. As can be seen from the figure, the adopted value $C$ III] $\lambda 1909 / C$ IV $\lambda 1549 \sim 0.1$ for gas at 8 lt-days implies that the density is high. $\left(\mathrm{C}^{2+}\right.$ must be present in this gas, since the fact that Ly $\alpha$ varies with the continuum shows that a $\mathrm{H}^{+}-\mathrm{H}_{0}$ ionization front is present.) At very much higher densities, line thermalization becomes increasingly more important, and normally weak lines such as C III $\lambda .977$ and $\mathrm{Al}$ III $\lambda 1860$ become strong. These lines are in fact observed in some objects (Osterbrock 1991), suggesting that the density in these objects may be much higher than we deduce for NGC 5548.

Some other lines (i.e., the Si IV line) are quite strong at intermediate densities. These lines depend on the chemical composition of the gas, unlike the $\lambda 1909 / \lambda 1549$ ratio, which depends only on ionization and density. The chemical composition of the emitting gas in NGC 5548 is essentially unknown, so we give by far the greatest weight to producing a $\lambda 1909 / \lambda 1549$ ratio $\sim 0.1$.

We adopt a density of $10^{11} \mathrm{~cm}^{-3}$, a compromise between suppression of the $\mathrm{C}$ III] line, and not making other, normally weak lines, too strong.

The ratio of radiation to gas pressure is also shown in Figure 2. The internally generated line radiation pressure is basically proportional to the intensity of the incident continuum, which is held fixed. The gas pressure increases with the hydrogen density (the temperature of the gas is constant to zeroth order), and the ratio of radiation to gas pressure falls below unity at densities slightly below $10^{11} \mathrm{~cm}^{-3}$. The density which we adopt, $N_{\mathrm{H}}=10^{11} \mathrm{~cm}^{-3}$, is also the lowest density which is stable against disruption by radiation pressure.

Finally, for our adopted continuum and density, the ioniza-

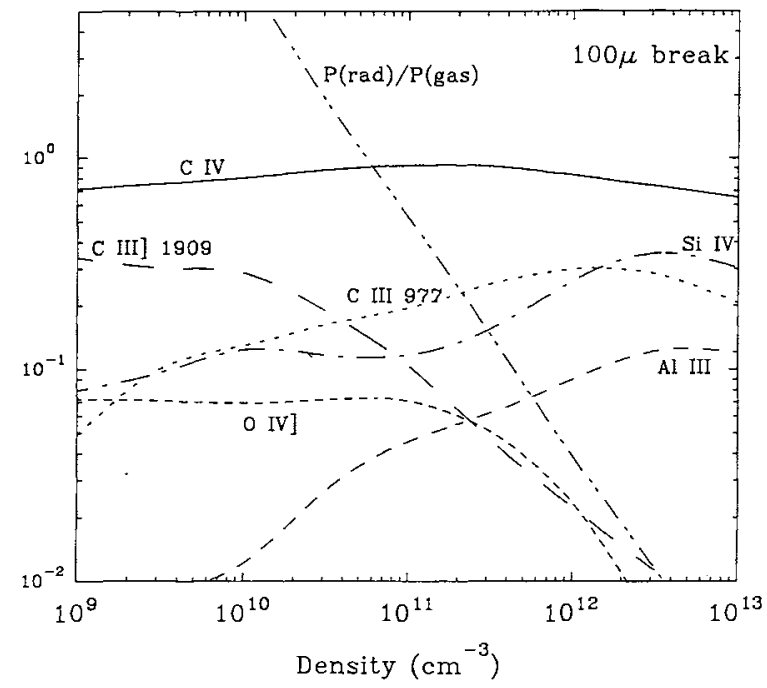

FIG. 2.-Emission-line intensities and radiation pressure are shown as a function of the gas density. The calculations are for constant density clouds with a Lyman limit optical depth of $10^{4}$. Lines are expressed as intensities relative to $\mathrm{Ly} \alpha$. The submillimeter break is assumed to occur at $100 \mu \mathrm{m}$. The limit of C III] $21909 / \mathrm{C}$ IV $21549<0.1$ corresponds to a density of $N_{\mathrm{H}} \geq 10^{11}$ $\mathrm{cm}^{-3}$. The ratio of the radiation to gas pressure is also shown and falls below unity at nearly the same density. 
tion parameter is $\log (U)=-0.96$. In the following we will adopt a density of $10^{11} \mathrm{~cm}^{-3}$ and an ionization parameter of $\log (U)=-1$ as our reference parameters.

\subsection{Dependence on the Ionization Parameter}

Having adopted a density in the neighborhood of $N \approx 10^{11}$ $\mathrm{cm}^{-3}$ and an ionization parameter $\log (U) \approx-1$, we now examine how observed line ratios depend on $U$. This is of special interest, since, if the source-cloud separation and cloud density remain constant, changes in the luminosity of the ionizing continuum correspond to changes in the ionization parameter. Plane-parallel constant baryon density clouds with column density $10^{26} \mathrm{~cm}^{-2}$, which is effectively an infinite optical depth in the ionizing continuum, were computed. The intensities (i.e., the sum of the inward and outward intensities) of several of the stronger lines relative to Ly $\alpha$ are shown in Figure 3.

Several trends are obvious. $\mathrm{H} \beta$ decreases in relative intensity because of the increased line optical depth (see below). High excitation lines ( $\mathrm{C}$ III $\lambda 977, \mathrm{O} v \lambda 1218$, and $\mathrm{O}$ vI $\lambda 1034$ ) all increase relative to $\mathrm{Ly} \alpha$ because the gas grows hotter and more highly ionized as $U$ increases. The line marked " $Q(\mathbf{H}) 1216$ " is the ratio of the number of $\mathrm{H}$-ionizing photons to the number of Ly $\alpha$ photons, which should have a value of unity if each ionizing photon creates one Ly $\alpha$ recombination. Ly $\alpha$ has an intensity substantially below its simple case $B$ value for large ionization parameters. This is because the line becomes a less effective radiator when the Ly $\alpha$ optical depth increases and line thermalization becomes more important.

A surprising prediction is the great strength of the $\mathrm{O} v \lambda 1218$ line. It is blended with Ly $\alpha$ because of the Doppler width of the BLR and is predicted to have an intensity $\sim 10 \%$ to $\sim 30 \%$ of Ly $\alpha$. The presence of this line would make Ly $\alpha$ appear redshifted relative to unblended lines (it is nearly $500 \mathrm{~km} \mathrm{~s}^{-1}$ redward of $\operatorname{Ly} \alpha$ ) and could affect the inferred line response to

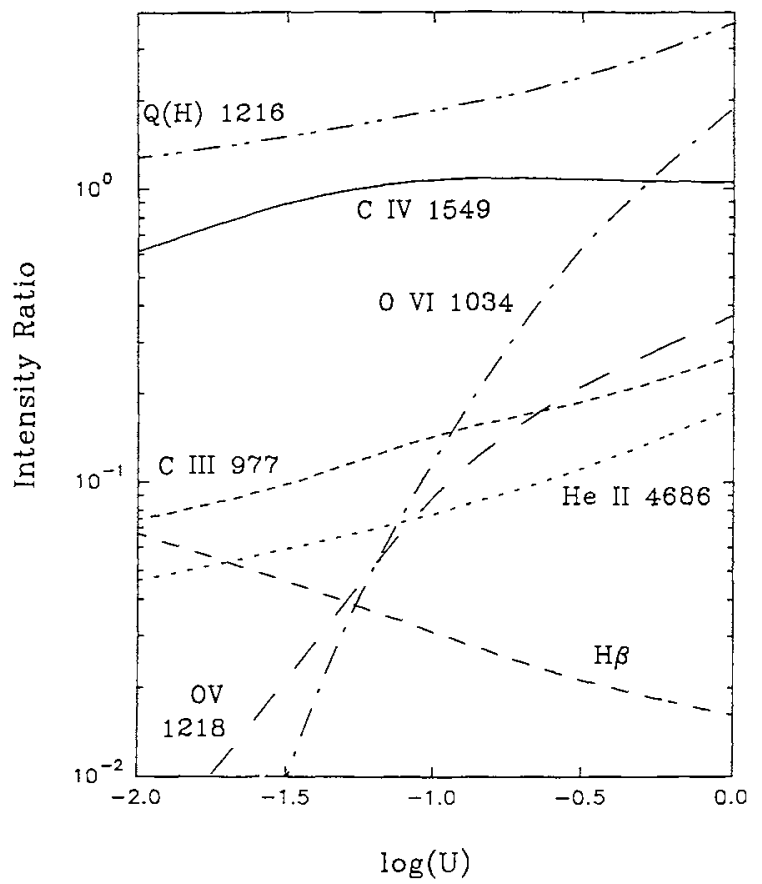

FIG. 3.- Line intensities relative to Ly $\alpha$ are shown as a function of the ionization parameter.

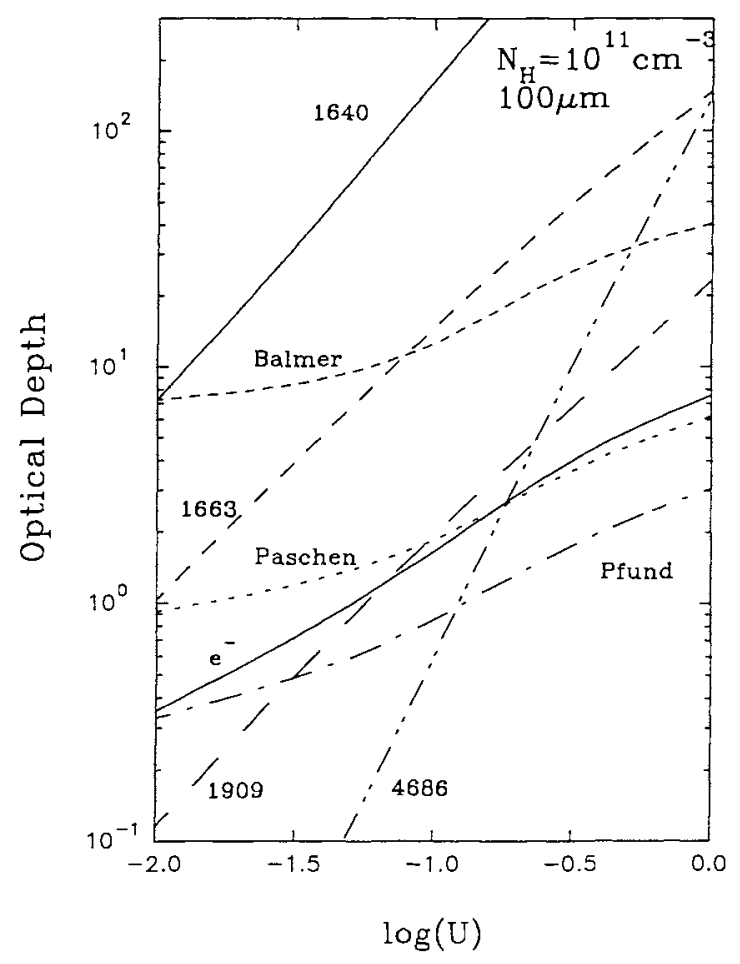

FIG. 4.-Line and continuum optical depths as a function of ionization parameter for gas with a density of $10^{11} \mathrm{~cm}^{-3}$. For the ionization parameter inferred for the C IV-Ly $\alpha$-emitting region of NGC $5548[\log (U)=-1]$, nearly all lines and continua are optically thick. Many of the lines shown, including $\mathrm{C}$ III] 21909, $\mathrm{O}$ III] 21663, and He II lines from excited states, are usually assumed to be optically thin. The optical depth for electron scattering is also shown. The BLR clouds at 8 lt-days are likely to be optically thick to electron scattering.

changes in the continuum. For instance, the present calculations predict that the $\mathrm{C}$ IV $\lambda 1549 / \mathrm{Ly} \alpha$ intensity ratio should not change as the ionization parameter increases, but the presence of strong $\mathrm{O} v$ emission would make the line ratio appear to decrease with increasing $U$.

\subsection{Line Optical Depths}

Optical depths in several lines and continua are shown as a function of ionization parameter in Figure 4.

It is clear from Figure 4 that, at high radiation densities, nearly all lines and continua are quite optically thick. The column density through the $\mathrm{H}^{+}$zone scales as $U c / \alpha$, where $\alpha$ is the hydrogen radiative recombination coefficient. Column densities in ions which are not dominant opacity sources increase with this $\mathrm{H}^{+}$column density, so the optical depth in most metal lines increases as $U$. The population of excited states is proportional to the flux of ionizing photons $\phi(\mathrm{H})=U N_{\mathrm{H}} c$ (Ferland, Netzer, \& Shields 1979), so the population and optical depths of excited states also increase. At very high ionization parameters, excited states of helium become optically thick. Figure 4 shows that He II $\lambda 4686$ has substantial optical depth; if these values of $U$ are relevant, then the He II $\lambda 4686 /$ $\lambda 1640$ intensity ratio, a commonly used reddening indicator, will not have its case B value. Finally, there is a substantial optical depth to electron scattering for most ionization parameters shown. Non-Doppler line broadening can occur in these cases.

Also, at a given column density, there will be an ionization 
parameter $U_{\text {ion }}$ for which the cloud is fully ionized:

$$
N_{\mathrm{H}^{+}} d l \approx U_{\text {ion }} \frac{c}{\alpha} .
$$

This expression is approximate since, for the intense radiation fields occurring here, the population of hydrogen in excited states is large, and the Balmer, Paschen, and higher continua are also effective in ionizing the gas. The spectrum of the cloud would change only gradually with increasing ionization parameter, until the ionization parameter reaches this critical value. Then, the cloud is fully ionized, and species which occur in the more neutral (shielded) regions of the cloud are diminished. When this occurs, the spectrum of the cloud changes dramatically; this is the reason that the $\mathrm{C}$ III/C IV flux ratio changes in Mushotzky \& Ferland (1984) at $\log (U) \approx-0.5$. (The clouds considered by Mushotzky \& Ferland had only a modest column density, $N_{\mathrm{H}} d l=10^{22.8} \mathrm{~cm}^{-2}$.)

\subsection{Emission-line Asymmetries}

Optically thick emission lines tend to be emitted from the illuminated face of the cloud; in the case of $\mathrm{Ly} \alpha$, essentially $100 \%$ of the line is emitted here (Ferland \& Netzer 1979). As a result of this, observers will see only clouds on the far side of an ensemble, even one which is spherically symmetric.

Here we compute the ratio of inward (i.e., toward the source of ionizing radiation) to total line intensities. The escape probability formalism described in the appendices, including destruction by the background continuum, is used. Figures 5 , 6 , and 7 show this ratio for the cases of $\operatorname{Ly} \alpha, \mathrm{H} \beta$, and $\mathrm{C}$ IV $\lambda 1549$, respectively. These are the best observed emission lines in NGC 5548.

The plots show the two parameters, the ionization parameter and column density, which are free parameters, given our assumptions. We do not vary the continuum shape, gas density, or chemical composition, since these do not affect the line beaming function, which is the aim of these calculations. The C Iv-Ly $\alpha$ clouds in NGC 5548 have $\log (U)=-1$, given a straightforward interpretation of the variability data. Figure 5

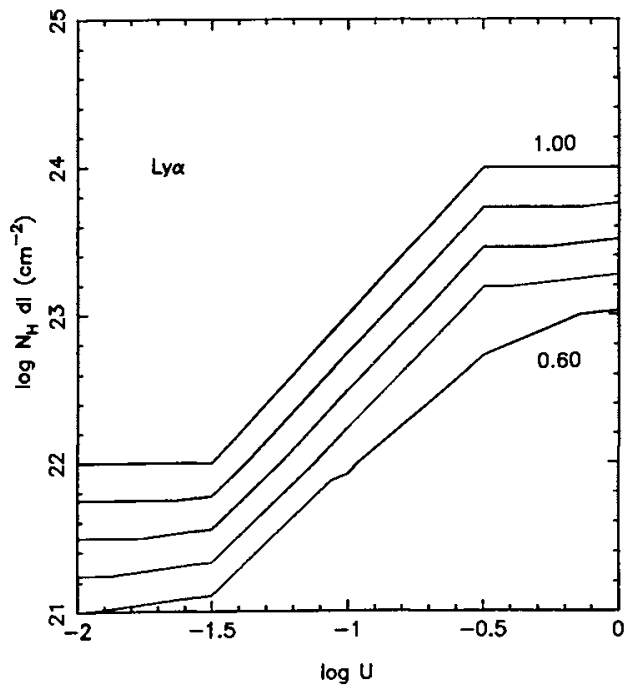

Fig. 5.- The contours show the fraction of the Ly $\alpha$ line emission which is emitted from the inner face of a model BLR cloud as a function of ionization parameter $U$ and total hydrogen column density $N_{\mathrm{H}} d l$. The contours are equally spaced at intervals of 0.10 .

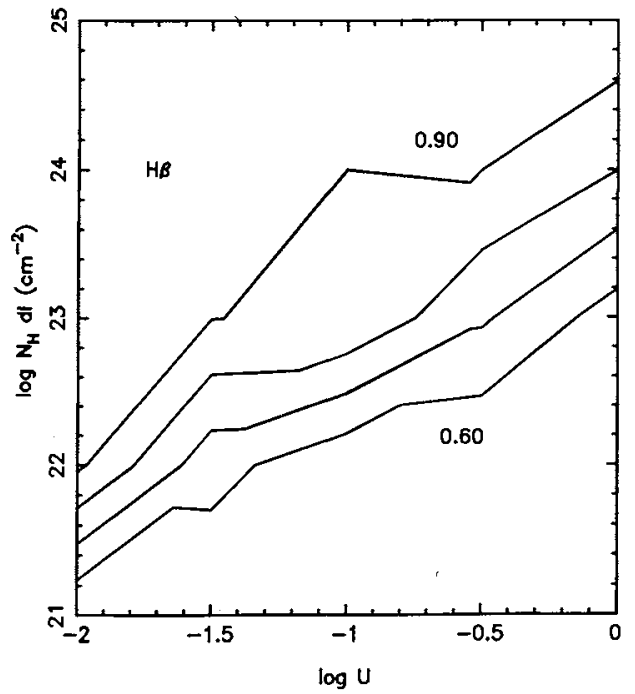

FIG. 6.-Fraction of $\mathrm{H} \beta$ emitted from the cloud inner face, plotted as in Fig. 5, with equally spaced contour intervals of 0.10 .

shows that the Ly $\alpha$ beaming ratio is near unity for most combinations of column density and ionization parameter. The large $U$-small column density combination of parameters do produce more uniform emission, because these clouds are fully ionized. Because of this, these clouds will be essentially invisible to variability studies (they respond only weakly to changes in the incident continuum). Only combinations of ionization parameter and column density which have strongly beamed Ly $\alpha$ need be considered here.

Beaming also occurs, but to a lesser extent, for $\mathrm{H} \beta$. For combinations of parameters where a hydrogen ionization front is present, we find a ratio of inward to total $\mathrm{H} \beta$ in the range $\sim 0.9$. C Iv $\lambda 1549$ is less beamed; typically $70 \%$ of the line is emitted in the inward direction. Most of the difference in the relative amount of beaming of $\mathrm{H} \beta$ and $\mathrm{C}$ iv is due to the higher Balmer line opacity through the partially ionized zone beyond the ionization front.

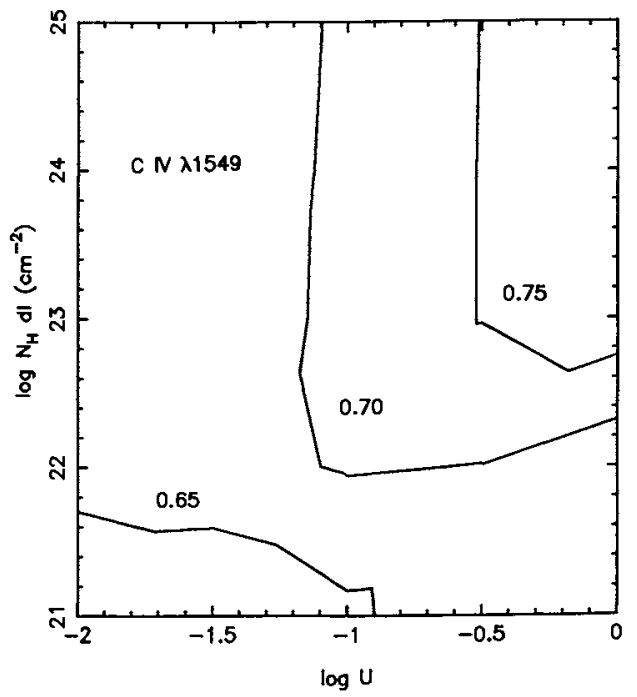

Fig. 7.-Fraction of C IV 21549 emitted from the cloud inner face, plotted as in Fig. 5 , with equally spaced contour intervals of 0.05 . 


\section{COMPARISON WITH OBSERVATIONS}

The response of a broad emission line changes in the continuum level can be described in terms of a transfer equation

$$
L(v, t)=\int_{-\infty}^{\infty} \Psi(v, \tau) C(t-\tau) d \tau,
$$

where $L(v, t)$ is the emission-line flux at time $t$ in the radial velocity range $v$ to $v+\mathrm{d} v, C(t)$ is the continuum light curve, and $\Psi(v, \tau)$ is the transfer function which describes the responsivity distribution of the line-emitting region as seen by an external observer (Blandford \& McKee 1982).

To understand how anisotropic emissivity affects the emission-line velocity profile and transfer function, it can be helpful to consider a thin spherical shell of radius $R$ through which flows line-emitting material with an outward velocity $V(R)$. Let $\theta$ be the angular coordinate around the shell measured from the point farthest away from the observer. Line emission excited by the central source of ionizing radiation will arive after a time delay given by

$$
\tau(R, \theta)=R(1+\cos \theta) / c,
$$

and with a line-of-sight velocity given by

$$
v(R, \theta)=V(R) \cos \theta \text {. }
$$

Since both the time delay and radial velocity of a given cloud are linear functions of $\cos \theta$, the spherical shell maps into a diagonal line in the velocity-delay $(v, \tau)$ plane, with $\cos \theta$ increasing uniformly along the diagonal line between the front point at $[-V(R), 0]$ and the back point at $[+V(R), 2 R / c]$. The element of surface area on the shell is $2 \pi R^{2} \sin \theta d \theta$, which is proportional to $d(\cos \theta)$. Thus the transfer function and velocity profile are found by simply projecting the diagonal line onto the $\tau$ and $v$ axes, respectively. For isotropic emission, the projection of the diagonal line yields the familiar boxcar forms of the transfer function and line profile.

For anisotropic emission, elements along the diagonal line

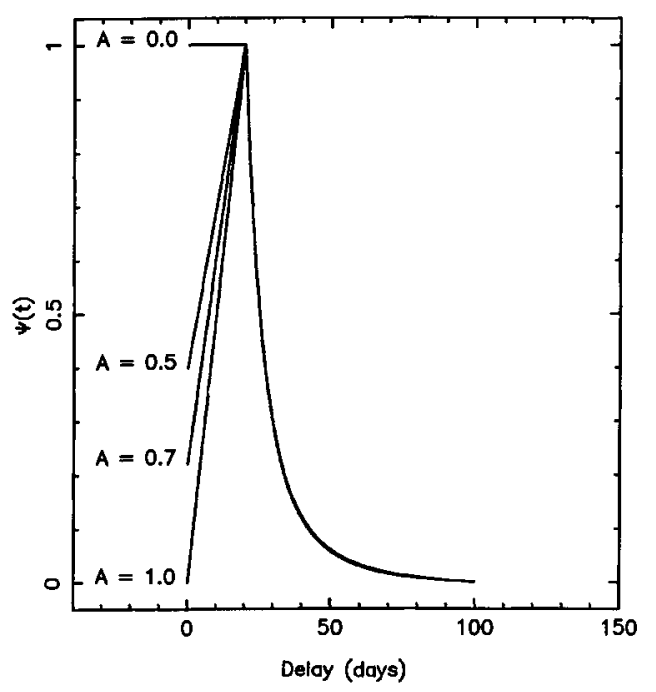

FIG. $8 a$ must be weighted by the emissivity as a function of $\cos \theta$ before projecting onto the axes. The form of anisotropic emission depends on the shapes of BLR clouds and is very model dependent. We adopt the following convenient parameterization of the anisotropy,

$$
I(\theta)=1+A \cos \theta,
$$

in which the ratio of inner face (toward the continuum source) to outer face (away from the continuum source) emissivity is $(1+A) /(1-A)$. This form of anisotropy is linear in $\cos \theta$; hence, the transfer function and velocity profile are also linear functions running from $1-A$ at the near side of the BLR, i.e., $(v, \tau)=[-V(R), 0]$, to $1+A$ at the far side of the BLR, i.e., $(v, \tau)=[+V(R), 2 R / c]$. The anisotropy shifts the mean velocity from 0 to $V(R)(A / 3)$, and the mean time delay is shifted from $R / c$ to $(1+A / 3) R / c$.

A general expression for the transfer function in the case of asymmetric emission is derived for the case of a spherically symmetric distribution in Appendix C. Transfer functions for several different values of $A$ are shown in Figure $8 a$. The case $A=0$ represents isotropically emitting clouds, and for $A=1$ the clouds do not emit at all in the direction away from the center. For the examples shown in Figure $8 a$ the volume emissivity of the ensemble of BLR clouds is assumed to vary with radius as a power law with index $\beta$ (see eq. [28]). The inner radius is set at $R_{\min }=10 \mathrm{lt}$-days, which produces a change in slope and often a peak in the transfer function at a delay of 20 lt-days, i.e., at twice the inner radius. The power-law index controls the rate at which the transfer function decreases to zero at longer delays. For the steep power-law index we consider here, $\beta=5$, the transfer function is relatively insensitive to the outer radius $R_{\max }$, which was set to 50 lt-days.

Figure $8 b$ shows the observed $\mathrm{H} \beta$ transfer function for NGC 5548 as found by Horne, Welsh, \& Peterson (1991). We consider the $\mathbf{H} \beta$ transfer function specifically because its behavior near zero delay is better determined than the behavior of the $\mathrm{Ly} \alpha$ or C IV transfer functions; this is because the $\mathrm{H} \beta$ lag is

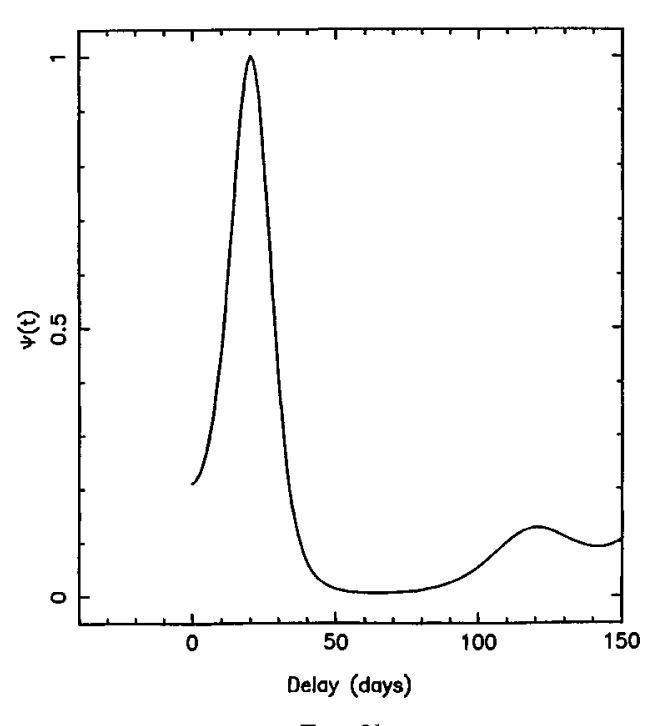

FIG. $8 b$

Fig. 8.-Panel (a) shows transfer functions for a spherical BLR (inner radius $10 \mathrm{lt}$-days, outer radius $50 \mathrm{lt}$-days) with various combinations of isotropic and anisotropic components of the line emission. The fraction of the line emission which is anisotropic is indicated, with $A$ as defined in eq. (8). Panel (b) shows the observed H $\beta$ transfer function for NGC 5548 from Horne, Welsh, \& Peterson (1991). In each case, the transfer functions have been renormalized to a peak value of unity. 
larger and the optical light curves have higher frequency (although less regular) sampling than do the UV light curves. The observed transfer function in Figure $8 b$ may be compared with the model transfer functions for spherically symmetric BLRs shown in Figure $8 a$.

The isotropic model $(A=0)$ gives a transfer function which is flat from 0 to $201 \mathrm{l}$-days and then declines to zero at $2 R_{\max } / c$. This model is not consistent with the observed transfer function because it has too much emission at time delays close to 0 . However, the anisotropic models, $(0<A \leq 1)$, give transfer functions that rise to a peak at $20 \mathrm{lt}$-days. The transfer functions with $A>0.7$ are reasonably compatible with the observed transfer function. Thus we have found a spherically symmetric but anisotropic model which is qualitatively consistent with the observed $\mathrm{H} \beta$ transfer function in NGC 5548.

If the BLR clouds do indeed radiate isotropically in the $\mathrm{H} \beta$ line, then the observed transfer function argues for a nonspherical geometry, such as a disk seen nearly face-on. However, we have shown that if the line emission is anisotropic, as we expect on the basis of physical arguments, the observed transfer function is consistent with a spherical distribution of BLR clouds.

\section{DISCUSSION}

We have shown that, for conditions now thought to exist in the BLR, essentially all lines are beamed back toward the source of ionizing radiation. This solves an old conundrum; it has long been known that Ly $\alpha$ is predominantly emitted from the illuminated face of the cloud (Ferland \& Netzer 1979; Ferland et al. 1979). If Ly $\alpha$ is beamed, but other lines emitted isotropically, then the fact that line profiles are, to low order, similar, presents a puzzle (see, for example, Baldwin \& Netzer 1978; Rees, Netzer, \& Ferland 1989). If, as we argue, all lines have a similar (predominantly inward) beaming function, then the first-order similarity of the various line profiles follows trivially.
We have also shown that a spherically symmetric geometry is consistent with the observed time lags, especially the lack of a strong signal arising from line-of-sight material. It does not, of course, follow that the geometry must be spherically symmetric. The issue of the BLR geometry is central to models of the origin of activity in AGN; if the BLR is an accretion disk surrounding a supermassive black hole (e.g., Dumont \& Collin-Souffrin 1989, and references therein) then a decidedly nonspherical geometry must be present.

It is important to keep all alternatives open, however. We have shown that spherical geometry is possible. This would be expected, for example, if BLR clouds are the extended atmospheres of or "comet-tails" behind stars (Edwards 1980; Mathews 1983). The idea has not been pursued with vigor since, at the time it was first proposed, the parameters thought to characterize the BLR, in particular the continuum flux at the BLR radius, did not seem to work out. However, the current estimate of the conditions within the BLR are entirely consistent with this picture.

Similarly, our calculations show that BLR clouds are likely to be optically thick to electron scattering. Electron scattering as a line-broadening mechanism has not been taken seriously since the early days of AGN investigations. This issue, like that of mass loss from stars, should be reconsidered.

The authors are grateful for support of this work by the National Science Foundation under grants AST 90-19692 (G. J. F.) and AST 89-15258 (B. M. P.) and by NASA under grants NAG 5-1366 (B. M. P. and G. J. F.) and NAG 5-1644 (S. N. N.) to The Ohio State University and under grants NAGW1796 and NAGW-2264 (K. H.) to Space Telescope Science Institute. The comments of Dan Maoz, Anil Pradhan, and David Hummer are gratefully acknowledged, and Richard Pogge is thanked for help with computer software.

\section{APPENDIX A}

\section{HYDROGENIC RATE COEFFICIENTS}

This Appendix describes the calculation of the hydrogenic recombination and recombination cooling rate coefficients needed in the present calculations.

As the density of a BLR cloud increases, line thermalization and continuum optical depths both increase. As a result, continuum processes are increasingly important as cooling mechanisms for the gas. In fact, at very high densities $\left(N>10^{13} \mathrm{~cm}{ }^{-3}\right)$ the clouds are primarily continuum radiators (Rees et al. 1989).

Figure 9 shows the thermal and ionization structure of the "standard" cloud deduced in the test. The ionization parameter is $\log (U)=-1$, and the density is $10^{11} \mathrm{~cm}^{-3}$. The upper panel shows the temperature structure, the middle panel shows the fraction of the total cooling which is carried by hydrogen recombination, and the lower panel shows the ionization structure of hydrogen and helium. At large depths in the cloud, where most lines are optically thick, continuum processes (mainly free-bound and free-free) are among the most important coolants. Even the continua, whose emission is the dominant coolant, are optically thick (Fig. 4). It is important that these continuum processes be treated with a precision commensurate with their importance.

In this section we derive state-specific hydrogenic recombination and recombination cooling coefficients over a wide range of temperatures.

\section{A1. FORMALISM}

The Milne relation for the state-specific radiative recombination rate coefficient $\left(\mathrm{cm}^{3} \mathrm{~s}^{-1}\right)$ to a level $n$ can be expressed as (Brown \& Mathews 1970; Gould 1978; Mihalas 1978)

$$
\begin{aligned}
\alpha(T, n) & =\left(\frac{2 \pi m k}{h^{2}}\right)^{-3 / 2} \frac{8 \pi}{c^{2}} \frac{g_{n}}{g_{e} g_{\text {ion }}} T^{-3 / 2} \int_{h v_{0}}^{\infty} v^{2} \sigma_{v}(n) \exp \left[\frac{-h\left(v-v_{0}\right)}{k T}\right] d v \\
& =4.12373 \times 10^{11} \frac{g_{n}}{g_{e} g_{\text {ion }}} T^{-3 / 2} \int_{h v_{0}}^{\infty} v^{2} \sigma_{v}(n) \exp \left[\frac{-h\left(v-v_{0}\right)}{k T}\right] d v_{\mathrm{Ryd}},
\end{aligned}
$$




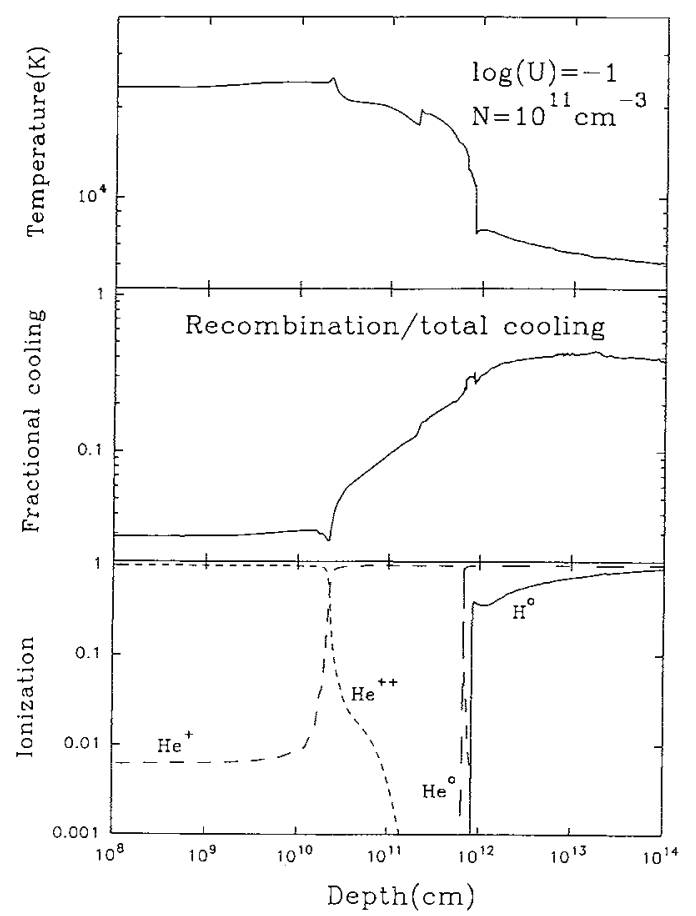

Fig. 9. - Structure of cloud with $\log (U)=-1$ and $N_{\mathrm{H}}=10^{11} \mathrm{~cm}^{-3}$. Hydrogen recombination cooling is a dominant coolant for regions deep in the cloud. The transport methods and state-specific recombination cooling coefficients described in the text are used.

where the $g$ 's are the statistical weights of the constituents, $h v_{\mathrm{Ryd}}$ is the photon energy in Rydbergs, $h v_{0} \approx z^{2} / n^{2}$ is the ionization potential in Rydbergs, $\sigma_{v}(n)$ is the photoionization cross section, and the other symbols have their usual meanings.

In implementing this formalism we explicitly took into account the fact that, for hydrogen, the energy scale is shifted by the ratio of the reduced mass of the nucleus to an infinite mass. If the energy of level $n$ of hydrogen is $n^{-2} R_{\mathrm{H}}$, then the coefficient appearing in the exponential is 157807 , not the commonly quoted 157890 . This does affect the results slightly since the energy scale enters as an exponential in equation (9).

Hydrogenic photoionization cross sections are required over a very wide range of energy since we wish to compute recombination coefficients over a wide range of temperature. Cross sections were calculated using a program based on routines developed by Hummer (1988) and Storey \& Hummer (1991, and private communication). The program generates the cross section values at arbitrary photon energies for all hydrogenic $(n, l)$ states, as well as for the total $n$, employing analytic expressions and some very accurate expansions and numerical procedures. The calculations were carried out at a number of different mesh sizes to check for convergence. We estimate that the results are typically accurate to better than $0.1 \%$.

The recombination cooling rate coefficient (ergs $\mathrm{cm}^{3} \mathrm{~s}^{-1}$ ) is given by

$$
k T \beta(T, n)=\left(\frac{2 \pi m k}{h^{2}}\right)^{-3 / 2} \frac{8 \pi}{c^{2}} \frac{g_{n}}{g_{e} g_{\text {ion }}} T^{-3 / 2} \int_{h v_{0}}^{\infty} v^{2} \sigma_{v}(n) h\left(v-v_{0}\right) \exp \left[\frac{-h\left(v-v_{0}\right)}{k T}\right] g v .
$$

Again, the integration is carried out numerically.

\section{A2. RESULTS}

The numerical results are presented in Tables 1 and 2. The first column of the table gives the log of the temperature; the next six columns give the total recombination coefficient $1 \leq n \leq 6$ summed over $l$ states. The next three columns give the total recombination coefficient for levels $7-10,11-20$, and $21-1000$, respectively. The last column gives the case $\mathrm{B}$ sum, $2 \leq n \leq 1000$. We consider a very large temperature range for completeness; at very low temperatures, three-body recombination predominates for most densities (Bates et al. 1962), while at very high temperatures other processes (i.e., Compton scattering, collisions) dominate the balance and the neutral fraction is vanishingly small.

As tests, we have compared our predictions of the recombination rate coefficients with those of Seaton (1959), Ferland (1980), Hummer \& Storey (1987), and Martin (1988). (Note that the total recombination rate given by Hummer \& Storey is the sum of radiative and net three-body recombination. For this comparison we used their results for a density of $10^{2} \mathrm{~cm}^{-3}$ to minimize the contribution of the second process.) The agreement with all of these results is good, usually much better than $1 \%$. Seaton (1959) presents the only calculation of recombination cooling coefficients of which we are aware. The present results agree with his to better than $5 \%$. Figure 10 shows the recombination cooling coefficient for several states.

Previous calculations were typically limited to temperatures encountered in Galactic gaseous nebulae, generally in the neighborhood of $10^{4} \mathrm{~K}$. We know of no calculations which extend over the temperature range we consider. However, it is possible to check that our results do approach two asymptotic limits. For very low temperatures the photoionization cross section is nearly constant 
TABLE 1

State-Specific and Case B Recombination COEFficients ${ }^{a}$

\begin{tabular}{|c|c|c|c|c|c|c|c|c|c|c|}
\hline $\log \left(T_{e}\right)$ & 1 & 2 & 3 & 4 & 5 & 6 & $7-10$ & $11-20$ & $21-$ & Case B \\
\hline 0.5 . & $9.258-12$ & $5.087-12$ & $3.512-12$ & $2.684-12$ & $2.172-12$ & $1.825-12$ & $5.303-12$ & $7.495-$ & 2.950 & $5.758-11$ \\
\hline 1.0 . & $5.206-12$ & $2.860-12$ & $1.974-1$ & $1.508-$ & $1.220-1$ & $1.025-12$ & $2.974-$ & 4.176 & 1.33 & $2.909-11$ \\
\hline 1.5. & $2.927-12$ & $1.608-12$ & $1.109-12$ & $8.465-13$ & $6.842-13$ & $5.737-13$ & $1.658-12$ & $2.285-12$ & $5.638-12$ & $1.440-11$ \\
\hline 2.0 & $1.646-12$ & $9.028-$ & $6.216-$ & $4.732-$ & $3.811-13$ & 3.183 & $9.081-13$ & $1.193-12$ & $2.173-$ & $6.971-12$ \\
\hline 2.5 & $9.246-13$ & $5.055-13$ & $3.460-13$ & $2.613-13$ & $2.084-13$ & $1.720-13$ & $4.753-12$ & $5.645-13$ & $7.476-13$ & $3.282-12$ \\
\hline 3.0 & 4. & 30 & & 1 & $1.085-1$ & 8.717 & $2.200-13$ & 2.31 & 3 & $1.489-12$ \\
\hline 3.5 . & $2.890-13$ & $517-13$ & $9.799-14$ & $6.884-1$ & $5.099-14$ & $3.912-14$ & $9.264-14$ & $8.016-1$ & $6.177-14$ & $6.430-13$ \\
\hline 4.0 & $2-13$ & $7.699-14$ & $.555-14$ & $2.965-1$ & $2.053-14$ & $1.487-14$ & $3.203-14$ & $2.381-14$ & $1.535-14$ & $2.588-13$ \\
\hline 4.5 . & $55-14$ & $461-$ & $1.812-14$ & $076-$ & 6.953 & 4.775 & 9.49 & 6.27 & 3.5 & 9.45 \\
\hline $5 .($ & 4 & 16 & 15 & 3 & 2.022 & & & & & \\
\hline 5.5 & $1.545-14$ & $196-15$ & $1.736-15$ & 8.9 & $5.219-16$ & 3.335 & $5.999-16$ & 3.444 & 1.6 & $8.793-15$ \\
\hline 6.0 & $5.058-15$ & $146-15$ & $392-16$ & $2.160-16$ & $1.229-16$ & $7.694-17$ & $1.347-16$ & 7.434 & $3.510-17$ & $2.245-15$ \\
\hline 6.5 . & 15 & 2.760 & 16 & 17 & $2.685-17$ & $1.660-17$ & 2.85 & $1.535-17$ & -18 & 5.19 \\
\hline & 16 & 6 & & & & & & & & \\
\hline 7.5 . & $7.006-17$ & $1.227-$ & $1-18$ & $1.976-$ & $1.083-18$ & $6.606-$ & 1.115 & 5.836 & 2.709 & $2.221-17$ \\
\hline 8.0 & $1.398-17$ & $2.377-1$ & $139-19$ & $2.759-19$ & $2.052-19$ & 1.248 & 2.097 & 1.090 & 5.179 & $4.267-18$ \\
\hline 8.5 & $2.665-18$ & 455 & & $6.970-$ & & & & 2.000 & 9.74 & 7.96 \\
\hline & & & & 1. & & & & & & \\
\hline 9.5 & $9.001-20$ & $1.481-20$ & $5.005-21$ & 2.294 & $1.247-21$ & $7.552-$ & $1.263-21$ & 6.518 & $3.382-22$ & $2.636-20$ \\
\hline $10.0 .$. & $1.623-20$ & $2.662-21$ & $8.985-22$ & $4.116-22$ & $2.235-22$ & $1.354-22$ & $2.263-22$ & $1.167-22$ & $6.267-23$ & $4.737-21$ \\
\hline
\end{tabular}

a The entry $9.258-12$ indicates a rate coefficient of $9.258 \times 10^{-12} \mathrm{~cm}^{3} \mathrm{~s}^{-1}$.

over the range where the exponential in equation (9) is large. In this case the integration is trivial, with the result

$$
\alpha \approx 2.6 \times 10^{6} n^{-2} T^{-1 / 2} \sigma_{n},
$$

where $\sigma_{n}$ is the cross section at threshold. The results go to this limit.

At very high temperatures the bandwidth in the integral is large, so $v^{-3}$ is a good approximation for the energy dependence of the cross section. The rate coefficient can then be expressed as

$$
\alpha \approx 4.1 \times 10^{11} n^{-4} T^{-3 / 2} \sigma_{n} \exp \left(h v_{0} / k T\right) E_{1}\left(h v_{0} / k T\right) .
$$

The results also go to this limit, to within a factor of 2 .

TABLE 2

State-Specific and Case B Recombination CoOling Coefficientsa

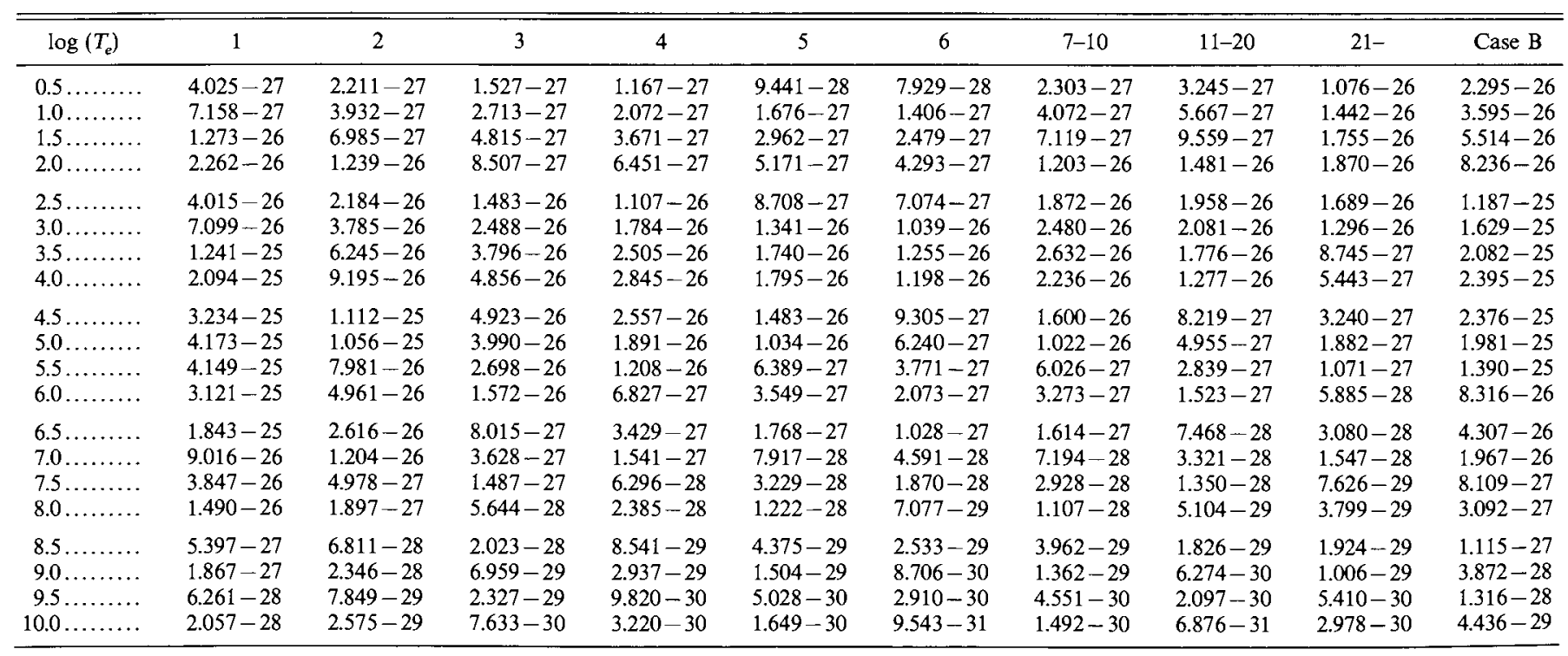

${ }^{a}$ The entry $4.025-27$ indicates a rate coefficient of $4.025 \times 10^{-27} \mathrm{ergs} \mathrm{cm}^{3} \mathrm{~s}^{-1}$. 


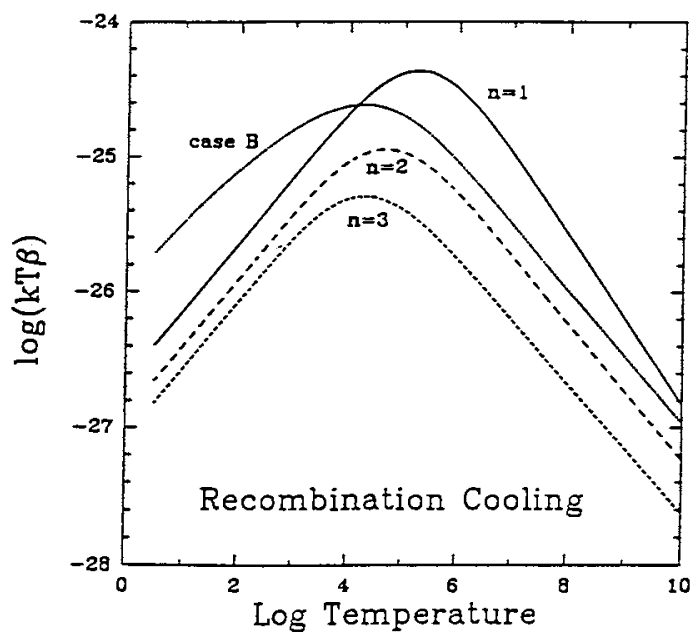

Fig. 10.-Recombination cooling coefficient. The product $k T \beta$ is shown as a function of temperature.

\section{A3. RATIONAL APPROXIMATIONS}

It is not numerically expedient to compute these rate coefficients on the fly in large-scale ionization-thermal structure calculations. We have fitted the rate coefficients with a high-order rational approximation. The rate coefficient is expressed as

$$
\alpha(T, n)=10^{F(T, n)} T^{-1}
$$

with

$$
F(T, n)=\frac{a_{n}+c_{n} x+e_{n} x^{2}+g_{n} x^{3}+i_{n} x^{4}}{1+b_{n} x+d_{n} x^{2}+f_{n} x^{3}+h_{n} x^{4}}
$$

and $x \equiv \log (T)$. The coefficients are listed in Table 3. These approximations reproduce our results with a mean error well below $0.1 \%$. For levels below $n=20$ the largest error is also under $0.1 \%$, although errors as large as $1.4 \%$ occur for the highest sum at temperatures below $100 \mathrm{~K}$.

TABLE 3

EXPANSION FOR RECOMBINATION COEFFICIENTS

\begin{tabular}{cccccccccc}
\hline \hline$n$ & $\mathrm{a}$ & $\mathrm{b}$ & $\mathrm{c}$ & $\mathrm{d}$ & $\mathrm{e}$ & $\mathrm{f}$ & $\mathrm{g}$ & $\mathrm{h}$ & $\mathrm{i}$ \\
\hline $1 \ldots \ldots$ & -10.7815 & -0.388896 & 4.68469 & 0.0640438 & -0.874235 & $-5.10248-03$ & 0.081411 & $2.47761-04$ & $-3.87713-03$ \\
$2 \ldots \ldots$ & -11.0434 & -0.393514 & 4.84334 & 0.0692118 & -0.956856 & $-5.49930-03$ & 0.092159 & $3.06273-04$ & $-5.02483-03$ \\
$3 \ldots \ldots$ & -11.2031 & -0.424576 & 5.25370 & 0.0819237 & -1.126131 & $-6.81708-03$ & 0.114221 & $4.11653-04$ & $-6.77511-03$ \\
$4 \ldots \ldots$ & -11.3145 & -0.439393 & 5.45398 & 0.0853755 & -1.166601 & $-7.40060-03$ & 0.118057 & $4.03664-04$ & $-6.48848-03$ \\
$5 \ldots \ldots$ & -11.4062 & -0.436467 & 5.46028 & 0.0863856 & -1.184309 & $-7.37118-03$ & 0.119145 & $4.12342-04$ & $-6.75160-03$ \\
$6 \ldots \ldots$ & -11.4846 & -0.442457 & 5.56951 & 0.0899765 & -1.241026 & $-7.54870-03$ & 0.125443 & $4.48713-04$ & $-7.53579-03$ \\
$7 \ldots \ldots$ & -11.0213 & -0.442776 & 5.37057 & 0.0943866 & -1.252699 & $-7.44688-03$ & 0.125435 & $4.88629-04$ & $-8.30273-03$ \\
$8 \ldots \ldots$ & -10.8751 & -0.450383 & 5.39988 & 0.1022072 & -1.341072 & $-7.17252-03$ & 0.129801 & $5.52029-04$ & $-9.92535-03$ \\
$9 \ldots \ldots$ & -10.2096 & -0.048286 & 0.85490 & 0.0453455 & -0.474956 & $-1.01818-03$ & 0.0 & 0.0 & 0.0 \\
\hline
\end{tabular}

TABLE 4

EXPANSION FOR RECOMBINATION COOLING COEFFICIENTS

\begin{tabular}{cccccccccc}
\hline \hline$n$ & $\mathrm{a}$ & $\mathrm{b}$ & $\mathrm{c}$ & $\mathrm{d}$ & $\mathrm{e}$ & $\mathrm{f}$ & $\mathrm{g}$ & $\mathrm{h}$ & $\mathrm{i}$ \\
\hline $1 \ldots \ldots$ & -26.6447 & -0.405110 & 11.2923 & 0.0672574 & -1.991084 & $-5.08026-03$ & 0.166268 & $2.05287-04$ & $-7.13575-03$ \\
$2 \ldots \ldots$ & -26.9067 & -0.416447 & 11.7104 & 0.0763838 & -2.268984 & $-5.84929-03$ & 0.196781 & $2.75885-04$ & $-9.96306-03$ \\
$3 \ldots \ldots$ & -27.0620 & -0.458344 & 12.8931 & 0.0899256 & -2.651639 & $-7.48544-03$ & 0.242675 & $3.39807-04$ & $-1.17865-02$ \\
$4 \ldots \ldots$ & -27.1827 & -0.491379 & 13.8557 & 0.1022522 & -3.023330 & $-8.56775-03$ & 0.282237 & $4.14455-04$ & $-1.46965-02$ \\
$5 \ldots \ldots$ & -27.2784 & -0.519318 & 14.6735 & 0.1110161 & -3.294623 & $-9.30673-03$ & 0.310205 & $4.64233-04$ & $-1.67032-02$ \\
$\ldots \ldots \ldots$ & -27.3596 & -0.549712 & 15.5609 & 0.1195189 & -3.566337 & $-9.84556-03$ & 0.335160 & $5.12181-04$ & $-1.88737-02$ \\
$7 \ldots \ldots$ & -26.9011 & -0.562123 & 15.6545 & 0.1207284 & -3.563036 & $-9.75175-03$ & 0.332549 & $5.16213-04$ & $-1.91249-02$ \\
$8 \ldots \ldots$ & -26.7582 & -0.536972 & 14.9208 & 0.1093810 & -3.253752 & $-8.32192-03$ & 0.293805 & $4.56629-04$ & $-1.75719-02$ \\
$9 \ldots \ldots$ & -26.1477 & -0.467188 & 12.6143 & 0.0821541 & -2.411906 & $-8.17803-03$ & 0.272395 & $5.99744-04$ & $-2.00967-02$ \\
\hline
\end{tabular}


Recombination cooling coefficients were fitted to equations of the form

$$
k T \beta(T, n)=10^{F(T, n)},
$$

where $F(T, n)$ is given above, and the fitting coefficients are listed in Table 4 . The errors in fitting these coefficients is larger, typically $0.5 \%$, but sometimes as large as several percent.

\section{APPENDIX B}

\section{NUMERICAL DETAILS}

This Appendix describes some of the numerical details in the calculations presented in the paper. Version 79 of the radiative equilibrium code "Cloudy" (Ferland 1991) is used in this work.

\section{B1. ESCAPE PROBABILITIES WITH BACKGROUND OPACITY}

This section describes recent improvements in the treatment of the escape probability function.

\section{B1.1. The Escape Probability Function}

For lines broadened by both thermal and microturbulent motions, the Doppler width is

$$
v_{\mathrm{D}}=\left(\frac{2 k T}{m}+v_{\mathrm{mic}}^{2}\right)^{1 / 2}
$$

where $m$ and $v_{\text {mic }}$ are the mass of the atom and microturbulent velocity, respectively. The mean line opacity is then

$$
\kappa_{l}=\frac{\pi e^{2} f}{m c \Delta v_{\mathrm{D}}}\left(N_{l}-N_{u} \frac{g_{l}}{g_{u}}\right),
$$

where $\Delta v_{\mathrm{D}}=v_{\mathrm{D}} v_{0} / c$ and $N_{l}$ and $N_{u}$ are the number densities $\left(\mathrm{cm}^{-3}\right)$ of the lower and upper states with statistical weight $g_{i}$, and the other symbols have their usual meanings (Osterbrock 1988).

The ratio of continuous to line opacity is parameterized as

$$
\beta=\frac{\kappa_{c}}{\kappa_{l}+\kappa_{c}}
$$

Line transfer can be described by either complete or partial redistribution. Which case applies is determined by the detailed processes affecting the level populations and line transport. In this work the lines are divided into two groups: strong resonance lines which are treated with partial redistribution with a Voigt profile, and subordinate lines, which are treated with complete redistribution in a Doppler core.

\section{B1.2. Resonance Lines}

The escape and destruction probability functions used for H I, He I, and He II Ly $\alpha$ and strong resonance lines such as $\mathrm{C}$ IV $\lambda 1549$ is that described by Hummer \& Kunasz (1980). Their tabulated values were fitted by interpolation.

\section{B1.3. Subordinate Lines}

Lines arising from excited states (hydrogen Balmer, Paschen, etc.) are treated assuming complete redistribution in a Doppler core (i.e., the damping constant $a$ is assumed to be zero). In this case, if the total optical depth of the slab is $T$, then the escape probability at a depth $\tau$ from the illuminated face is given by

$$
P(\tau, T, \beta)=[1-\beta F(\beta)] \frac{1}{2}\left[K_{2}(\tau, \beta)+K_{2}(T-\tau, \beta)\right]
$$

and the destruction probability is

$$
D(\beta)=\beta F(\beta)
$$

The function

$$
F(\beta)=\int_{-\infty}^{\infty} \frac{\phi(x)}{\beta+\phi(x)} d x,
$$

where $\phi(x) \approx \pi^{-1 / 2} \exp \left(-x^{2}\right)$ is the Voigt function, is interpolated from the tables presented by Hummer (1988). The function

$$
K_{2}(\tau, \beta) \equiv \frac{1}{1-\beta F(\beta)} \int_{-\infty}^{\infty} \frac{\phi^{2}(x)}{\beta+\phi(x)} E_{2}\{[\beta+\phi(x)] \tau\} d x
$$

is evaluated numerically. 


\section{B1.4. Level Populations}

The radiative line de-excitation rate is given by

$$
\Lambda_{u l}=\left(\frac{d N_{u}}{d t}\right)_{\mathrm{rad}}=N_{u} A_{u, l}(P+D)-N_{l} A_{u, l} \eta \gamma_{u, l},
$$

where $\eta$ is the photon occupation number of the external radiation field and $\gamma_{u, l}$ is the escape probability described by Castor (1970). The local cooling rate due to the line is related to the volume emissivity of the line by

$$
\Lambda_{u l}=4 \pi j(h v)=N_{u} A_{u, l} P(\tau, T, \beta) h v=\left(N_{l} C_{l, u}-N_{u} C_{u, l}\right) h v,
$$

where $C_{i, j}$ are elements of the cascade matrix (Osterbrock 1988), and the local flux (cm ${ }^{-2} \mathrm{~s}^{-1}$ ) of "on-the-spot" (OTS) photons caused by line loss (used to compute heating or photoionization rates for the sources of the background opacity) is

$$
\phi_{\mathrm{OTS}}=\frac{N_{u} A_{u, l} D(\beta)}{\kappa_{c}} .
$$

The ratio of inward to total line intensity is then given by

$$
\frac{4 \pi j(\text { in })}{4 \pi j(\text { total })}=\frac{K_{2}(\tau, \beta)}{\left[K_{2}(\tau, \beta)+K_{2}(T-\tau, \beta)\right]}
$$

\section{B2. THE HYDROGEN ATOM}

The present treatment of the hydrogen atom, and its interaction with the external radiation field, largely follows that described by Ferland \& Rees (1988).

Several improvements in the treatment of the far-infrared-radio continuum. The free-free heating rate is now calculated exactly, by integrating over the attenuated incident continuum, rather than by approximating the far-infrared continuum as a power law. This is done by dividing the energy interval $\sim 7 \times 10^{-7} \mathrm{eV}$ to $100 \mathrm{keV}$ into $\sim 400$ cells with roughly constant $\delta v / v$. This also allows the photoionization and induced recombination rates for quantum levels $3 \leq n \leq 7$, along with their respective heating and cooling rates, to be computed by integrating over the attenuated incident continuum, without approximation. These changes allow the code to better treat environments which are optically thick at the relevant energies.

The formalism outlined above is used to treat the escape and local photoionization of all 30 hydrogen lines explicitly computed. All sources of background opacity (mainly free-free, $\mathrm{H}^{-}, \mathrm{H}$, and $\mathrm{He}$ bound free-opacities) are included as line destruction processes, and OTS flux corresponding to each line is included as ionizing or heating sources for the relevant opacity sources.

\section{B3. RECOMBINATION CONTINUUM ESCAPE PROBABILITIES}

Recombination to level $n_{0}$ produces radiation capable of ionizing levels $n \geq n_{0}$. The transport of this radiation can be treated with variations of the OTS approximation (Van Blerkom \& Hummer 1967; Davidson \& Netzer 1979; Netzer \& Ferland 1984; Osterbrock 1988; Bassgen, Bassgen, \& Grewing 1988). If hydrogen is the dominant opacity and the photoelectric cross section varies as $\left(v / v_{0}\right)^{-3}$, then the probability of escape of a photon is given by

$$
P_{\text {esc }}\left(\tau, \frac{h v_{0}}{k T}\right)=\frac{\int_{1}^{\infty} x^{-1} E_{2}\left(\tau_{0} x^{-3}\right) \exp \left[-h v_{0} / k T(x-1)\right] d x}{\int_{1}^{\infty} x^{-1} \exp \left[-h v_{0} / k T(x-1)\right] d x},
$$

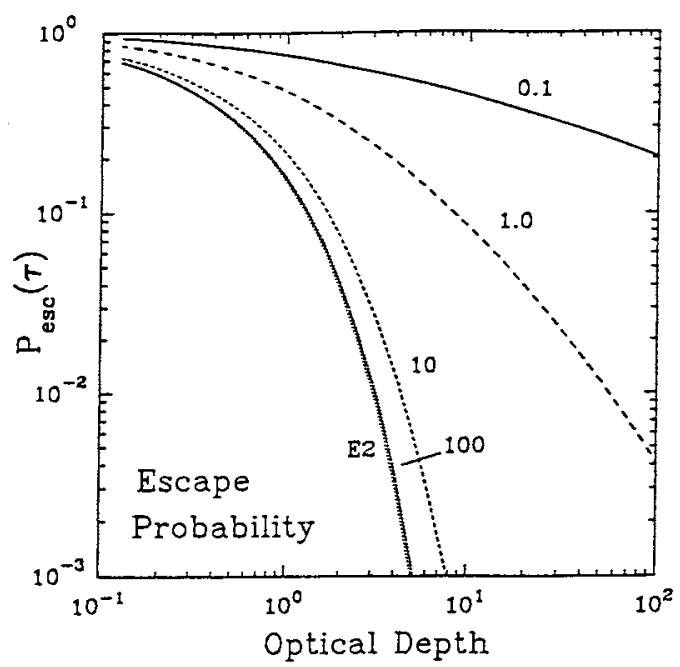

FIG. 11.-Continuum escape probability function. The continuum escape probability is shown as a function of the optical depth at threshold, for a variety of

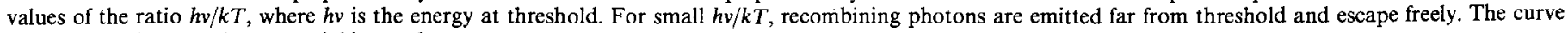
labeled $E_{2}$ is the second exponential integral. 
where $E_{2}$ designates the second exponential integral; $\tau_{0}$ is the optical depth at threshold, which has energy $h v_{0} ;$ and $x \equiv h v / h v_{0}$. No simple approximation is obvious, and the function is integrated numerically.

Figure 11 shows $P_{\text {esc }}$. The escape probability decreases as the optical depth increases in the expected nearly exponential manner. It decreases with increasing $h v_{0} / k T$. Physically, as the gas temperature increases relative to the threshold energy, recombining photons are created further from threshold, where the optical depth of the gas is smaller. For small $h v / k T$ and large optical depths, the escape probability is several orders of magnitude larger than that suggested by the optical depth at threshold alone (curve labeled $E_{2}$ ). For large $h v / k T$, the escape probability is nearly $E_{2}(\tau)$, as expected. The use of this geometric escape probability to modify the recombination rate coefficient and determine an OTS flux of ionizing photons is described in Baldwin et al. (1991).

\section{APPENDIX C}

\section{DERIVATION OF THE TRANSFER FUNCTION}

In this Appendix, analytic expressions for the transfer function are derived for the case of anisotropic emission.

Let $R_{\min }$ and $R_{\max }$ denote, respectively, the inner and outer radii of a spherical BLR, and let $\mu=\cos \theta$, where $\theta$ is the angle between a cloud and the point on the back of the BLR farthest away from the observer, as measured from the center of the BLR $(0 \leq \theta \leq \pi)$. Let $\beta$ denote the power-law index of the volume emissivity and $A$ the anisotropy factor $(0 \leq A \leq 1)$, i.e.,

$$
F(r, \mu)=F_{0}\left(\frac{r}{R_{\min }}\right)^{-\beta}(1+A \mu) .
$$

The time delay $\tau$ is defined as the delay between the arrival of a direct pulse and its echo $\left(0 \leq \tau \leq 2 R_{\max } / c\right)$, and so

$$
\tau=\frac{r}{c}(1+\mu)
$$

The response of the BLR gas contained in a volume $d V$ at location $\mu, r$ is then

$$
\Psi(\mu, r) d \mu d r=2 \pi r^{2} d r d \mu F_{0}\left(\frac{r}{R_{\min }}\right)^{-\beta}(1+A \mu) .
$$

Now $\Psi(\mu, r) d \mu d r=\Psi(\tau, r) d \tau d r$, so using equation (29) to replace $\mu$ and $d \mu$ with expressions containing $\tau$ we get

$$
\Psi(\tau)=\int_{R_{0}}^{R_{\max }} \Psi(\tau, r) d r=\int_{R_{0}}^{R_{\max }} 2 \pi r c F_{0}\left(\frac{r}{R_{\min }}\right)^{-\beta}\left[(1-A)+\frac{A c \tau}{r}\right] d r,
$$

where $R_{0}$ is the radius of the innermost shell that contributes to $\Psi$ at the time delay $\tau$, i.e., $R_{0}$ is the larger of $R_{\min }$ and $c \tau / 2$. The above expression can be simplified to

$$
\Psi(\tau)=(1-A) \Psi_{1}+\tau A \Psi_{2}
$$

where

$$
\begin{aligned}
\Psi_{1} & =2 \pi c F_{0} R_{\min }^{2}\left(\frac{1}{\beta-2}\right)\left[\left(\frac{R_{0}}{R_{\min }}\right)^{-(\beta-2)}-\left(\frac{R_{\max }}{R_{\min }}\right)^{-(\beta-2)}\right] \quad(\beta \neq 2) \\
& =2 \pi c F_{0} R_{\min }^{2} \ln \left(\frac{R_{\max }}{R_{0}}\right) \quad(\beta=2)
\end{aligned}
$$

and

$$
\begin{aligned}
\Psi_{2} & =2 \pi c^{2} F_{0} R_{\min }\left(\frac{1}{\beta-1}\right)\left[\left(\frac{R_{0}}{R_{\min }}\right)^{-(\beta-1)}-\left(\frac{R_{\max }}{R_{\min }}\right)^{-(\beta-1)}\right] \quad(\beta \neq 1) \\
& =2 \pi c^{2} F_{0} R_{\min } \ln \left(\frac{R_{\max }}{R_{0}}\right) \quad(\beta=1) .
\end{aligned}
$$

For $\tau \leq 2 R_{\min } / c, R_{0}=R_{\min }$ and so $\Psi_{1}$ and $\Psi_{2}$ are independent of $\tau$. Equation (32) is thus a simple linear function of $\tau$. Note that if $A=0$ (no anisotropy), then $\Psi(\tau)=\Psi_{1}$, which is independent of $\tau$. At a time delay of zero, $\Psi(\tau=0)=(1-A) \Psi_{1}$, which falls from $\Psi_{1}$ when there is no anisotropy, to zero when the anisotropy is complete. For sufficiently large values of $\beta$, the peak of $\Psi$ occurs at $\tau_{\text {peak }}=\left(2 R_{\min } / c\right)$ and ranges in value from $\Psi_{1}$ to $\tau_{\text {peak }} \Psi_{2}$ as $A$ ranges from 0 to 1 .

For $\tau \geq 2 R_{\min } / c, R_{0}=\mathrm{c} \tau / 2$ and $\Psi_{1}$ and $\Psi_{2}$ are no longer independent of $\tau$. In this case we have (for $\beta \neq 1$ and $\beta \neq 2$ )

$$
\Psi\left(\tau \geq \frac{2 R_{\min }}{c}\right)=C_{1} A \tau^{-(\beta-2)}+C_{2}(1-A) \tau^{-(\beta-2)}-C_{3} A \tau-C_{4}(1-A),
$$

where the values $C_{n}$ are independent of $\tau$. 


\section{REFERENCES}

Baldwin, J. A., Ferland, G. J., Martin, P. G., Corbin, M. R., Cota, S. A. Peterson, B. M., \& Slettebak, A. 1991, ApJ, 374, 580

Baldwin, J. A., \& Netzer, H. 1978, ApJ, 226, 1

III Bassgen, G., Bassgen, M., \& Grewing, M. 1988, A\&A, 200, 51

$\rightarrow$ Bates, D. R., Kingston, A. E., \& McWhiter, R. W. P. 1962, Proc. Roy. Soc. Lond. A, 267, 297

Blandford, R. D., \& McKee, C. F. 1982, ApJ, 255, 419

Brown, R. L., \& Mathews, W. G. 1970, ApJ, 160, 939

Castor, J. I. 1970, MNRAS, 149, 111

Clavel, J., et al. 1991, ApJ, 366, 64

Davidson, K., \& Netzer, H. 1979, Rev. Mod. Phys., 51, 715

Dumont, A. M., \& Collin-Souffrin, S. 1989, A\&AS, 83, 71

Edwards, A. C. 1980, MNRAS, 190, 757

Ferland, G. J. 1980, PASP, 92, 596 91-01.

Ferland, G. J., \& Netzer, H. 1979, ApJ, 229, 274

Ferland, G. J., Netzer, H., \& Shields, G. A. 1979, ApJ, 232, 382

Ferland, G. J., \& Persson, S. E. 1989, ApJ, 347, 656

Ferland, G. J., \& Rees, M. J. 1988, ApJ, 332, 141

Gould, R. J. 1978, ApJ, 219, 250.

Horne, K., Welsh, W. F., \& Peterson, B. M. 1991, ApJ, 367, L5

Hummer, D. G. 1988 , ApJ, 327, 477

Hummer, D. G., \& Kunasz, P. B. 1980, ApJ, 236, 609

Hummer, D. G., \& Storey, P. J. 1987, MNRAS, 224, 801

Krolik, J. H., Horne, K., Kallman, T. R., Malkan, M. A., Edelson, R. A., \& Kriss, G. A. 1991, ApJ, 371, 541

Martin, P. G. 1988, ApJS, 66, 125

Mathews, W. G. 1983, ApJ, 272, 390

Mathews, W. G., \& Ferland, G. J. 1987, ApJ, 323, 456

Mihalas, D. 1978, Stellar Atmospheres (2d ed; San Francisco: Freeman)

Mushotzky, R. F., \& Ferland, G. J. 1984, ApJ, 278, 558

Netzer, H., \& Ferland, G. J. 1984, PASP, 96, 593

Netzer, H., \& Maoz, D. 1990, ApJ, 365, L5

Osterbrock, D. E. 1988, Astrophysics of Gaseous Nebulae and Active Galactic Nuclei (Mill Valley: University Science Books)

Peterson, B. M. 1988, PASP, 100, 18

Peterson, B. M., et al. 1991, ApJ, 368, 119

Rees, M. J., Netzer, H., \& Ferland, G. J. 1989, ApJ, 347, 640

Seaton, M. J. 1959, MNRAS, 119, 81

Storey, P. J., \& Hummer, D. G. 1991, Comput. Phys. Comm., 66, 129

Turner, T. J., \& Pounds, K. A. 1989, MNRAS, 240, 833

Van Blerkom, D., \& Hummer, D. G. 1967, MNRAS, 137, 353 